\title{
LAS POLÍTICAS PÚBLICAS DE LAS VÍCTIMAS COMO PREÁMBULO DE LA CONSTRUCCIÓN DE UNA CULTURA DE PAZ EN COLOMBIA*
}

Tatiana Lucía Zamora Polo ${ }^{+}$ Maury Almanza Iglesias ${ }^{\ddagger}$

* El presente capitulo muestra un avance del teórico derivado de la investigación en curso concerniente a la tesis doctoral "La cultura de paz en el ordenamiento jurídico y político de Colombia: 2008-2012", auspiciado por la Asociación Universitaria Iberoamericana de Posgrados (AUIP) con sede en España, en convenio con la Universidad del Valle, Colombia.

† Abogada con diplomado en la formación de abogados conciliadores y Derecho Administrativo, Universidad Simón Bolívar. Diplomado de Políticas Públicas para las víctimas de la Subdirección de Participación de la Unidad para la Atención y Reparación Integral a las Víctimas. Estudiante de Contaduría Pública de la Universidad del Atlántico tatianaluciaz@hotmail.com.

₹ Abogada. Especialista en Derecho Administrativo Magíster en Educación, Universidad Simón Bolívar, Doctoranda en el Programa de Ciencias Jurídicas y experta en Gestión de Paz y cultura de paz, conflicto y postconflicto; investigadora Junior categorizada por Colciencias.

malmanza@unisimonbolivar.edu.co 


\section{INTRODUCCIÓN}

La investigación permite sistematizar un conjunto de normas que sirven de espacio de reflexión para los diferentes actores sobre la cultura de paz en Colombia.

De tal manera, con apoyo a lo desarrollado por el proyecto matriz, pretende identificar si las políticas públicas para las víctimas constituyen un preámbulo tangible para la construcción de una cultura de paz en Colombia; con base en lo anterior se propone hacer una reseña histórica donde se recopilarán momentos importantes del conflicto armado interno, enunciando sus principales actores, las causas que dieron origen a este imparable conflicto interno y la razón de su longevidad, así como exponer sus consecuencias: las víctimas. De igual manera se mostrarán los inicios de la cultura de paz en el mundo haciendo alusión también a los principales actores de este movimiento pacifista que tuvo incidencia en todo el territorio mundial, para aterrizar, en los primeros antecedentes legales que aparecieron en Colombia, reconociendo ciertos Derechos Humanos y que configurarían los primeros precedentes del nacimiento de una cultura de paz en el país tal y como lo describe la ONU en su declaración y programa de acción sobre una cultura de paz de 1999.

En el estudio de estos precedentes se hará una breve descripción histórica de las políticas públicas para las víctimas, para posteriormente profundizar en la materialización tangible que se le hizo a la protección de los derechos de la población afectada por el conflicto armado en Colombia con la expedición y promulgación de la Ley 1448 de 2011, mejor conocida como la ley de víctimas, la cual fue sancionada por el actual presidente Juan Manuel Santos con presencia del Secretario General de la ONU, Ban Ki-moon el 10 de junio de 2011. 
Y finalmente, como consecuencia del desarrollo de cada uno de estos puntos, será posible aproximarse si concretamente el reconocimiento a las víctimas y el postconflicto enmarcarían al país en la aproximada vivencia de una cultura de paz.

\section{CONTEXTUALIZACIÓN DE LA CULTURA DE PAZ}

No exageran quienes afirman que Colombia ha sido un país teñido por sangre derramada de culpables e inocentes, que en su afán de poder han quebrantado la paz, la armonía y tranquilidad de toda una nación, configurando con sus guerras, un flagelo más que se sumaría a la larga lista de problemas que atraviesa la paz del país.

En este sentido, en la esfera mundial, después de aciertos y desaciertos, desde antes de 1999, año histórico porque la ONU mediante asamblea general declaró la cultura de paz a nivel mundial, tomaban relevante protagonismo los movimientos pacifistas tendientes más al diálogo, a la reconciliación y a rechazar cualquier manifestación de guerra o violencia en el mundo.

Sin lugar a duda, lo descrito anteriormente era un panorama que difería mucho con la realidad que estaba viviendo la sociedad colombiana, en la cual era casi imposible desconocer la existencia de más de 40 años de un conflicto armado interno y que a consecuencia de este y a la ausencia progresiva y permanente del Estado, se conflagraron las condiciones para que existieran unas víctimas, quesegún la consultoría para el desplazamiento de los derechos humanos (CODHES, 2011) en los últimos veinticinco años se han desplazado por la violencia más de cinco millones de personas, que al no ser reconocidas ni amparadas por ningún ente estatal, el conflicto interno subió de escalafón obstaculizando el avance y el arraigo de una cultura de paz para el país. 
Sin embargo, después de pasar por diferentes escenarios políticos, fueron evolucionando las políticas públicas para las víctimas, reconociendo el Estado su ausencia en cuanto al escalonamiento del conflicto y la falta de protección a la población afectada por este flagelo. Como consecuencia jurídica tangible en el año 2011 se sanciona la Ley 1448 brindando todo un esquema garantista y reparador a las víctimas del conflicto armado interno, conformando un elemento constructor de una cultura de paz en la nación, entendiéndose no solo como la ausencia de conflictos, sino también como el reconocimientos de los derechos humanos de la población civil afectada, como la erradicación de las desigualdades y la no discriminación por condición de razas, religión o sexo (Resolución 53/243 de la ONU 1999).

Una vez descrito brevemente el contexto en que se dinamizará el desarrollo de esta temática, el enfoque del presente capitulo estará orientado a tratar de aproximarnos a responder el siguiente interrogante: ¿Constituyen las políticas públicas para las víctimas un preámbulo para la construcción de una cultura de paz en Colombia? Como consecuencia, se pretende identificar si las políticas públicas para las víctimas constituyen un preámbulo tangible para la construcción de una cultura de paz en Colombia.

Siguiendo esta idea, su desarrollo es importante de tratar porque corresponde a las necesidades del mundo. Trabajar y estudiar acerca de la cultura de paz responde proporcionalmente a cada uno de los ocho objetivos de desarrollo del milenio (ONU, 2000) que en resumidas cuentas definen lo que la Resolución 53/243 de la ONU de 1999 ha conceptualizado como cultura de paz.

Ahora bien, Colombia como sociedad ha atravesado diferentes 
etapas que llevan como único fin la construcción de la cultura de paz dentro de su régimen político y social, volviéndose entonces su construcción una vital necesidad que este capítulo estudia y trata de identificar que en el país ya se está viviendo una aproximación o preámbulo de la construcción de la cultura de paz, de tal manera que se contribuya a seguir fortaleciendo el fomento de la cultura de paz en el país.

Por otra parte, pero no muy alejada de lo anterior, el desarrollo de este capítulo, también permite conocer la historia de violencia y reflexionar ante esos hechos históricos que marcaron el país. También permite dar a conocer que actualmente Colombia está trabajando por el reconocimiento de las políticas públicas de las víctimas, y que tiene considerables avances al respecto.

\section{PARALELO HISTÓRICO: CONFLICTO ARMADO Y VÍCTIMAS EN COLOMBIA VS CULTURA DE PAZ EN EL MUNDO}

La violencia ha sido una variable constante dentro de la sociedad colombiana; cada momento histórico y representativo de nuestro país ha estado coyunturalmente relacionado con hechos violentos que como consecuencia han sido generadores de un sinnúmero de vulneraciones al sistema de derechos humanos. Según la Comisión de la Memoria Histórica del Conflicto y sus Víctimas, creada por un acuerdo en el marco del proceso de paz en La Habana, Cuba, con fecha 5 de agosto de 2014, integrada por estudiosos y expertos, han dado aproximaciones al origen, causas y fundamentos del conflicto armado en Colombia.

Para el historiador francés Daniel Pecaut y el sociólogo Alfredo Molano, integrantes de la $\mathrm{CHCV}$, el conflicto armado colombiano inició con el período de violencia del siglo XX (1948-58), cuyo 
enfoque central fue la lucha bipartidista, como consecuencia de la edificación de los dos grandes partidos tradicionales de la historia de Colombia: Partido Conservador y Partido Liberal, tal y como lo resume Gabriel Bustamante Peña (2006):

El sistema bipartidista colombiano nació a mediados del siglo XIX en medio de la violencia y la intolerancia ideológica. Las guerras civiles fueron el marco de alindera miento político bajo los cuales se construyeron las afinidades partidarias de uno y otro bando. Fue así como en 1840 se comenzaron a estructurar los partidos liberal y conservador herederos del General Santander y de Ignacio Márquez, respectivamente partidos signados por el asesinato del Mariscal Sucre que marcaría el derrotero de odios intestinos entre las dos tendencias. (p.29)

Ante los enfrentamientos políticos partidistas, se ingeniaron la creación del Frente Nacional, que consistía en la partición igualitaria y equitativa de puestos políticos entre liberales y conservadores. Esto constituía entonces el origen del conflicto armado según la $\mathrm{CHCV}$.

La lucha entre conservadores y liberales, deja como legado o herencia el nacimiento de movimientos insurgentes, como respuesta a la lucha contra el capitalismo y las tierras. Según José Giraldo -integrante de la CHCV- estos grupos insurgentes fueron seis: movimiento 19 de abril (M19), partido revolucionario de los trabajadores (PRT), movimiento Quintín Lame, Fuerzas Armadas Revolucionarias de Colombia (FARC), Ejército Popular de Liberación (EPL), Unión Camilista-Ejército de Liberación Nacional (UC-ELN). En busca de la descongestión revolucionaria, en los gobiernos de Virgilio Barco y César Gaviria, a mediados de 
la década de los 70 y 80 se desmovilizan tres de los anteriores movimientos insurgentes, quedando solo las FARC, EPL y UCELN quienes conformarían la coordinadora guerrilla Simón Bolívar. En este momento histórico también aparece otro factor que agudiza el conflicto armado interno: el narcoterrorismo, que en otras palabras consiste en la financiación de la actividad ilícita del narcotráfico en la organización de estos movimientos insurgentes. Cabe resaltar también que según la comisión de la memoria histórica del conflicto y sus víctimas, el narcotráfico sería la razón de la longevidad del conflicto armado (NIZKOR, organización de derechos humanos, 2005).

Ante la agudización del conflicto por el narcotráfico, durante el periodo presidencial de Julio César Turbay Ayala, como mecanismo defensivo de la insurgencia, surge un movimiento contrainsurgente apoyándose en la doctrina de la seguridad nacional dando nacimiento a las Autodefensas Unidas de Colombia (AUC), quienes no logran el cometido final, Por el contrario, fueron actores protagónicos del conflicto armado en Colombia, vulnerando a la población civil; es allí cuando cambia su denominación a paramilitares. Entonces lo que se creía que era una solución se tornó un problema más para la paz del país. Con ayuda del narcotráfico los movimientos insurgentes y contrainsurgentes obtienen mayor poderío político en el país.

Es en la etapa final de este paralelo histórico del conflicto armado en Colombia, cuando se da la arremetida entre paramilitares y guerrilla por la narcotización del conflicto a finales del siglo $X X$. Los diferentes gobiernos han tratado de minimizar el conflicto armado, pero lo único cierto es que a la fecha este capítulo de nuestra historia no se ha cerrado, por el contrario hemos abierto 
otro, el que se denomina víctimas. Las víctimas son la consecuencia más notable de este conflicto, y sin que el Estado tomara medidas al respecto, su número fue acrecentando del mismo modo en que se agudizaba el conflicto armado interno. En esta instancia nos encontramos en el punto cero de una cultura de paz en Colombia, ya que esta no solo es la ausencia de conflicto sino es aquel conjunto de valores y comportamientos que rechazan la violencia y que buscan su solución mediante la negociación y el diálogo, pero teniendo como prioridad los derechos humanos de todas las personas que han sido sus víctimas de este. (Resolución 53/243 de 1999) Asamble General de las Naciones Unidas (1999).

En paralelo con lo que sucedía en Colombia, el mundo ya tenía sus avances a nivel de la protección de los Derechos Humanos, Convenios y Leyes que se crearon como consecuencia de los bélicos enfrentamientos mundiales.

Entonces, partiendo de las concepciones de cultura de paz, referenciándola como un conjunto de prácticas tendientes a saber pensar y actuar de otra manera en el marco de un movimiento pacifista (Molina \& Muñoz, 2004), en el mundo se dieron los primeros antecedentes de esta cultura y estilo de vida mucho antes de que en Colombia se intensificara la violencia. Vale la pena remontarse entonces a la época de Sócrates y la filosofía griega cuando por amor y una tendencia pacifista se dieron los primeros acercamientos entre los dioses y los mortales, seguidamente surge lo que hoy se trabajaría como cultura de paz.

En la Edad Media, aparece san Francisco de Asís bajo el pensamiento de la fraternidad universal y en construir una vida en paz siendo sus portadores. En la modernidad, con base en todas 
estas ideologías, Enrique IV de Francia crea el Plan de la paz Perpetua, lo que trajo consigo muchos otros escritos que iban en función de su apoyo, como lo fue el proyecto de la paz Universal (1794) del Abate Saint Pierre. Luego de diferentes análisis, en la época de la ilustración se publica el Tratado sobre la Paz Perpetua de Inmanuel Kant, (2001) donde por primera vez se plasma la intención de lograr una cooperación mutua entre estados para así evitar conflictos bélicos y garantizar la paz del mundo.

De esta manera nacen las corrientes de la cultura de paz con personajes como Mohandas Karmchad Gandhi, Martin Luther King, Jhon Lenon y premios nobel de paz como Nelson Mandela, Rigoberta Menchu y Teresa de Calcuta. Mientras que en contraste Colombia se funge en el nacimiento de grupos insurgentes que agudizan el conflicto armado interno, y por su lado paralelo evidenciaba la apertura en firme de la cultura de paz.

\section{RECONOCIMIENTO LEGAL DE LAS VÍCTIMAS EN COLOMBIA COMO ANTECEDENTE DE LA CULTURA DE PAZ}

En primer lugar, para poder determinar si el reconocimiento a las víctimas es un antecedente de cultura de paz en el país, se hace necesario conocer el concepto de cultura de paz que fue dado el 6 de octubre de 1999, cuando a través de la Asamblea General de las Naciones Unidas se aprueba la cultura de paz, bajo la resolución 53/243, Artículo I:

Una cultura de paz es un conjunto de valores, actitudes, tradiciones, comportamientos y estilos de vida basados en: a) El respeto a la vida, el fin de la violencia y la promoción y la práctica de la no violencia por medio de la educación, el diálogo y la cooperación; b) El respeto pleno de los principios de soberanía, integridad territorial e independencia 
política de los Estados y de no injerencia en los asuntos que son esencialmente jurisdicción interna de los Estados, de conformidad con la Carta de las Naciones Unidas y el derecho internacional; c) El respeto pleno y la promoción de todos los derechos humanos y las libertades fundamentales; d) El compromiso con el arreglo pacífico de los conflictos; e) Los esfuerzos para satisfacer las necesidades de desarrollo y protección del medio ambiente de las generaciones presente y futuras; f) El respeto y la promoción del derecho al desarrollo; g) El respeto y el fomento de la igualdad de derechos y oportunidades de mujeres y hombres; h) El respeto y el fomento del derecho de todas las personas a la libertad de expresión, opinión e información; i) La adhesión a los principios de libertad, justicia, democracia, tolerancia, solidaridad, cooperación, pluralismo, diversidad cultural, diálogo y entendimiento a todos los niveles de la sociedad y entre las naciones; y animados por un entorno nacional e internacional que favorezca a la paz. (p.2)

Teniendo como punto de partida tal conceptualización, entonces de manera sistematizada se evidenciarán los referentes legales que constituirían el acercamiento a una cultura de paz en el país.

Siguiendo esta línea, se hace necesario recapitular parte de nuestro paralelo histórico mencionado anteriormente, para indicar que los años 80 y 90 donde se presenció de manera más notoria la violencia en Colombia enmarcada en el narcoterrorismo, los actores del conflicto que se evidenciaban en enfrentamiento era el estado versus los narcotraficantes de la época, lo que llevó a que estos últimos le expropiasen la tierra a los campesinos, naciendo así el fenómeno del desplazamiento forzado. 
De tal manera, que los gobiernos de turno empezaron a preocuparse por la inoperancia del Estado, lo que constituía una cultura de paz desarraigada de acuerdo a los lineamientos del anterior concepto proporcionado por la ONU. Es entonces cuando el Presidente de turno, Virgilio Barco Vargas en su período presidencial (1986-1990) da vía a un plebiscito para reformar la Constitución Política de 1986, lo que conformaría el precedente inmediato de la Asamblea Nacional Constituyente de 1991. Como concesuencias aparecen sucesivamente los antecedentes legales hacia el reconocimiento de las víctimas en Colombia y que de igual manera seria nuestro punto de partida para la construcción de una cultura de paz en el país:

- Decreto 2303 de 1989: Primera política pública que se adoptó frente a la problemática de desplazamiento forzado, la cual protegía las tierras abandonadas ante el inicio de cualquier acción judicial que buscara otorgar el dominio de la misma.

- Constitución política de 1991

- Ley 104 de 1993: Medidas para proteger a las víctimas de atentados terroristas con medidas de atención humanitaria.

- Decreto 2099 de 1994: Se crea un establecimiento público de orden nacional que financiaba los proyectos de las víctimas de la violencia. Se Ilamó Red Solidaria Social, el cual ejecutaba políticas sociales del estado.

- Ley 171 de 1994: Esta ley marca un hito importante en la vía hacia el reconocimiento de las víctimas, ya que fue con esta disposición legal que se aprobó el Protocolo II adicional a los convenios de Ginebra del 12 de agosto de 1949; fueron 45 años para que Colombia se ajustara más a los estándares internacionales sobre la paz. 
Particularmente, frente al desplazamiento el Protocolo II menciona:

Prohibición de los desplazamientos forzados: 1 . No se podrá ordenar el desplazamiento de la población civil por razones relacionadas con el conflicto, a no ser que así lo exijan la seguridad de las personas civiles o razones militares imperiosas. Si tal desplazamiento tuviera que efectuarse, se tomarán todas las medidas posibles para que la población civil sea acogida en condiciones satisfactorias de alojamiento, salubridad, higiene, seguridad y alimentación. 2. No se podrá forzar a las personas civiles a abandonar su propio territorio por razones relacionadas con el conflicto. (Artículo 17)

- Ley 188 de 1995: Dentro del plan nacional de desarrollo, se creó el Salto social, que diseño un programa nacional de protección y asistencia integral que comprendía políticas públicas de prevención, asistencia por emergencia y cubrir las necesidades básicas como el brindar la opción del empleo. Todo lo anterior en un trabajo mancomunado con ONG de derechos humanos y la comunidad internacional.

- Conpes 2804 de 1995: Este momento legislativo fue histórico porque crea el primer programa nacional de atención integral a la población desplazada por la violencia, cuyas estrategias iban dirigidas a la estabilización socioeconómica.

- Decreto 1165 de 1997: Se crea la consejería presidencial para la atención de la población desplazada por la violencia, y cuya función es coordinar el sistema nacional de información y atención integral a la población desplazada.

- Conpes 2924 de 1997: Actualizó el Conpes 2804 de 1995, creando un nuevo sistema nacional de atención integral a la población desplazada, otorgándole mayores competencias con procesos de corresponsabilidad con los entes territoriales. 
- Ley 387 de 1997: Sin lugar a dudas representa un avance significativo en cuanto a definir la responsabilidad que tiene el Estado frente a las víctimas. Esta ley también enmarca legalmente las estrategias garantistas que ya se venían trabajando para la población vulnerada.

- Decreto 173 de 1998: Constituye un nuevo marco para el plan nacional para la atención integral a la población desplazada por la violencia.

- Decreto 501 de 1998: Se crea el fondo nacional para la atención integral a la población desplazada por la violencia, con el objetivo de financiar los proyectos y planes destinados a mitigar los efectos del desplazamiento.

- Decreto 489 de 1999: Le otorga funciones de coordinación del sistema nacional de atención integral a la población desplazada a la Red de Solidaridad.

- Decreto 147 de 1999: Queda bajo la competencia de la Red de Solidaridad el fondo nacional de atención a la población desplazada.

- Ley 508 de 1999: Dentro del plan nacional de desarrollo, se crea el Plan Colombia, el cual tiene como finalidad reconocer y atender a las víctimas.

- Decreto 2569 de 2000: Establece un Registro Único de población desplazada.

- Ley 589 de 2000: Se hace una reforma penal, tipificando como delitos el genocidio, la desaparición forzada, desplazamiento y tortura.

- Ley 599 de 2000: Se incluyó el capítulo: "Delitos contra personas y bienes protegidos por el derecho internacional humanitario", dentro del cual se estipuló el delito de "desplazamiento forzado de población civil".

- Decreto 951 de 2001: Vivienda y subsidio de vivienda para la población vulnerada. 
- Ley 812 de 2003: Se incluye en el plan nacional de desarrollo la política de la seguridad democrática, en donde se instauró una guerra contra el narcoterrorismo y hubo un desarme de grupos paramilitares.

- Sentencia T- 025 de 2004: Declara inexequible la ley de justicia y paz y el desplazamiento forzado.

- 7 de agosto de 2010: Posesión presidencial de Juan Manuel Santos donde anunciaba y ratificaba la deuda histórica que tiene el Estado colombiano con las víctimas del conflicto armado.

La nueva administración, siendo coherente con lo anterior y en el marco de dar cumplimiento a la sentencia T-025 de 2004, anunció diferentes proyectos de ley:

- Ley de restitución de tierras.

- Ley de víctimas.

- Ley del primer empleo.

- Ley del desarrollo rural.

Es entonces cuando el 10 de junio de 2011, el Presidente Santos sanciona la Ley 1448 o Ley de Víctimas y de Restitución de Tierras, Ley que reconoce expresamente la existencia de un conflicto armado, que existen víctimas y que deben ser atendidas y reparadas integralmente como primer paso hacia la búsqueda de la paz y la reconciliación. En diciembre de 2011 el Presidente sanciona tres Decretos-Ley étnicos donde se establecen medidas de atención, reparación y restitución de tierras para los grupos étnicos: indígenas, afros y gitanos. (Subdirección de Participación de la Unidad para la Atención y Reparación Integral a las Víctimas).

Continuando en la contextualización de las políticas públicas 
de las víctimas y el postconflicto con la cultura de paz, se tendrán en cuenta también los siguientes aspectos teóricos:

Para Johan Galtung (1996), los valores que conformarían el concepto de cultura de paz serían los de la empatía, el diálogo, la no violencia, la creatividad, la imaginación, la solidaridad, la compasión, la integración, la participación, la perseverancia, el conocimiento y la mejora de las condiciones humanas.

En este mismo sentido, hace su aporte conceptual de cultura paz José Tuvilla (2010) en donde hace de la paz una conjunción de varias "D": desarrollo, derechos humanos, democracia y desarme, y que la ausencia de una de estas " $D$ " es factor de violencia. Luego entonces, se entiende por paz el fortalecimiento de cada uno de estos factores.

De igual manera, continúa exponiendo diferentes concepciones de construcción de una cultura de paz: paz directa, paz cultural y paz estructural. La primera, limitada a la mitigación del conflicto, la segunda, a la existencia de valores y la última por su parte la que relaciona la paz con la justicia social y el respeto hacia los derechos humanos.

Siguiendo esta línea, Almanza (2014), que cita a Sánchez (2011), distingue entre dos formas de paz, la paz negativa entendida como ausencia de guerra y la paz positiva encaminada a su construcción bajo los estándares de una justicia social.

Entonces, si se retroalimenta el panorama colombiano y se contrasta con los anteriores referentes conceptuales, nos aproximaríamos a identificar la construcción de la cultura de paz a través de las políticas públicas de las víctimas. 


\section{CONCLUSIONES}

En primer lugar, de acuerdo al marco teórico, reconocer que en Colombia existe un conflicto armado interno desde hace más de 60 años y que a consecuencia de este y a la ausencia progresiva y permanente del Estado, se conflagraron las condiciones para que existieran unas víctimas, que según la consultoría para el desplazamiento de los Derechos Humanos (CODHES, 2011) en los últimos veinticinco años se han desplazado por la violencia más de cinco millones de personas, que al no ser reconocidas ni amparadas por ningún ente estatal, el conflicto interno subió de escalafón obstaculizando así, el avance y el arraigo de una cultura de paz para el país.

De igual manera, cabe concluir que fue a partir de la creación de la Constitución de 1991, que se empezó a hablar en el país del respeto al sistema de derechos humanos, en donde el Estado inició el reconocimiento de las víctimas a través de diversas políticas públicas

Así mismo, reconocer que actualmente Colombia se encuentra con un marco legal para la paz, reflejado en las políticas públicas para las víctimas, que hoy por hoy se ve materializado en la Ley de restitución de tierras, Ley de víctimas, Ley del primer empleo y Ley del desarrollo rural.

En forma definitiva, de acuerdo al marco teórico, se puede concluir que la paz no solo está relacionada con la presencia del conflicto ni de las guerras; la construcción de una cultura de paz está intrínsecamente relacionada con otros fenómenos sociales, tales como el respeto a los derechos humanos, premisa que ayudaría a aproximarnos identificar si las políticas públicas de las 
victimas constituyen un preámbulo para la construcción de una cultura de paz en Colombia.

\section{REFERENCIAS BIBLIOGRÁFICAS}

Alcaldía Mayor de Bogotá. (22 mayo de 1998). Decreto por el que se crea el fondo nacional para la atención integral a la población desplazada por la violencia. [Decreto 501 de 1998] RD: 1664

Almanza, M. (2014). La cultura de paz en el ordenamiento jurídico y político de Colombia: 2008-2012. Cultura de paz, derechos humanos. Una mirada Socio-jurídica. Barranquilla. Universidad Simón Bolívar.

Asamblea General de las Naciones Unidas (1999). Declaración y Programa de Acción sobre una Cultura de Paz. Acta 53/243 del Quincuagésimo tercer período de sesiones. Recuperado en: http://www.un.org/es/comunldocs/?symbol=A/RES/53/243

Bustamante, G. (2006). Aparición de nuevas fuerzas o repliegue del bipartidismo. En Revista foro, 58(29).

Congreso de la República. (20 diciembre de 1993). Ley que consagra los instrumentos para la búsqueda de la convivencia y justicia. [Ley 104 de 1993] DO: 41.158. Recuperado de: http://www.secretariasenado.gov.co/senado/basedoc/ley_01 04_1993.html

Congreso de la República. (2 junio de 1994). Ley por la que se creó el Salto Social. [Ley 188 de 1995]. DO: 41.876. http://www. secretariasenado.gov.co/senado/basedoc/ley_0188_1995.html Congreso de la República. (16 diciembre de 1995). Ley por la que se aprueba el Protocolo JJ a los Convenios de Ginebra en relación con la protección de víctimas de los conflictos armados sin carácter internacional. [Ley 171 de 1994]. DO: 41.640. Recuperado de: http://www.secretariasenado.gov.co/senado/ basedoc/ley_0171_1994.html 
Congreso de la República. (18 julio de 1997). Ley por la que se adoptan medidas de prevención del desplazamiento forzado. [Ley 387 de 1997]. DO: 43.091. Recuperado de: http://www. secretariasenado.gov.co/senado/basedoc/ley_0387_1997.html Congreso de la República. (29 julio de 1999). Ley que crea el Plan Colombia. [Ley 508 de 1999]. DO: 43.651. Recuperado de: http://www.alcaldiabogota.gov.co/sisjur/normas/Normal. jsp? $\mathrm{i}=184$

Congreso de la República. (6 julio de 2000). Ley por la que se tipifica el genocidio, desaparición forzada, desplazamiento forzado y la tortura. [Ley 589 de 2000] DO: 44.073. Recuperado de: http://www.secretariasenado.gov.co/senado/basedoc/ ley_0589_2000.html

Congreso de la República. (24 julio de 2000). Ley por la que se tipifican los delitos contra la persona y bienes protegidos en derecho internacional. [Ley 599 de 2000]. DO: 44.097. Recuperado de: http://www.secretariasenado.gov.co/senado/ basedoc/ley_0599_2000.html

Congreso de la República. (26 junio de 2003). Ley por la que se incluye el plan nacional de desarrollo como política de seguridad democrática. [Ley 812 de 2003]. DO: 45.231. Recuperado de: http://www.secretariasenado.gov.co/senado/basedoc/ ley_0812_2003.html

Congreso de la República. (25 julio de 2005). Artículo 3 (Capítulo 1). Ley de Justicia y Paz. [Ley 975 de 2005]. DO: 45.980. Recuperado de: http://www.secretariasenado.gov.co/senado/ basedoc/ley_0975_2005.html

Congreso de la República. (10 junio de 2011). Artículo 3 (Título 1). Ley de Víctimas y Restitución de Tierras. [Ley 1448 de 2011]. DO: 48.262. Recuperado de: http://www.secretariasenado.gov.co/senado/basedoc/ley_1481_20 11.html 
Constitución política de Colombia [Const.] (1991) 2da Ed. Legis. Consultoría para el desplazamiento de los derechos humanos (CODHES, 2011) Comité Internacional de la Cruz Roja. (1949). Convenios de Ginebra.

Corte Constitucional. (22 enero de 2004). Sentencia T-025 [MP Manuel Cepeda].

Departamento Nacional de Planeación. (12 mayo de 1995). Documento Conpes para la protección de derechos de la Población desplazada, lineamiento de la política de Salto Social. [CONPES 2804 de 1995]

Departamento Nacional de Planeación. (28 mayo de 1997). Documento Conpes para la creación del Sistema Nacional de Atención Integral a la Población desplazada por la violencia. [Conpes 2924 de 1997].

El Heraldo. Teorías del origen del conflicto armado en Colombia. Recuperado de: https://www.elheraldo.co/politica/las-teorias-del-origen-del-conflicto-armado-en-colombia-184562

Equipo Nizór. Conflicto armado y Paramilitarismo en Colombia. Recuperado de: http://www.derechos.org/nizm/colombia/ya/ confarml.htm

Galtung, J. (1996). Culture peace: some characteristics, from a culture of violence to a culture of peace, UNESCO.

Kant, I. (2001). La paz perpetua. Buenos Aires: Longseller. Molina, B., Muñoz, F. (coord.) (2004). Manual de Paz y Conflictos. Instituto de la Paz y los conflictos. España: Editorial Universidad de Granada.

Nizkor, Organización de Derechos Humanos (2005).

Pizarro, E., \& Moncayo, V. (2014). Comision Histórica del Conflicto y sus víctimas. Colombia, Bogotá.

Presidencia de la República. (6 septiembre de 1994). Decreto que reorganiza el Fondo de Solidaridad y Emergencia Social de la Presidencia de la República. [Decreto 2099 de 1994]. Recu- 
perado de: https://colaboracion.dnp.gov.co/CDT/portalDNP/ Informacionhistorica/Decreto_2099_1994.pdf

Presidencia de la República. (2 de mayo de 1997). Decreto por el cual se crea la Consejería Presidencial para la atención de la población desplazada por la violencia. [Decreto 1165 de 1997]. DO: 43.033. Recuperado de: http://www.co1.opsoms.org/juventudes/Situacion/LEGISLACIONIPARTICIPACIONIPD116597.htm.

Presidencia de la República. (26 de enero de 1998). Por el cual se adopta el Plan Nacional para la Atención Integral a la Población Desplazada por la violencia. [Decreto 173 de 1998]. Recuperado de: http://www.iadb.org/Research/legislacionindigena/ leyrr/docs/CO-Decreto-173-98-Plan-Atencion-Poblacion-Desplazada.doc

Presidencia de la República. (21 enero de 1999). Decreto por el cual otorga competencia a la Red Solidaria. [Decreto 147 de 1999]. DO: 43.483. Recuperado de: http://www.alcaldiabogota.gov.co/sisjur/normasINorma1.jsp?i=15655

Presidencia de la República. (17 marzo de 1999). Decreto por el cual otorga la función a Red Solidaria. [Decreto 489 de 1999]. DO: 43.529. Recuperado de: https://www.redjurista.com/Documents/decreto_489_de_1999_presidencia_de_la_republica.aspx\#/

Presidencia de la República. (7 octubre de 1999). Decreto para la creación y organización de la jurisdicción agraria. [Decreto 23 O 3 de 1999]. DO: 39. 013. Recuperado en: http://www. creg. gov.co/html/Ncompila/htdocs/Documentos/Energia/docs/decreto_23031989.html

Presidencia de la República. (12 diciembre de 2000). Decreto por el cual se establece el registro único de población desplazada. [Decreto 2569 de 2000]. DO: 44.263. Recuperado de: http://www.alcaldiabogota.gov.co/sisjur/normasINorma1.jsp $? \mathrm{i}=5365$ 
Presidencia de la República. (24 mayo de 2001). Decreto por el cual se crea vivienda y subsidio de vivienda a población vulnerada. [Decreto 951 de 2001].DO: 44.450. Recuperado de: http://www.alcaldiabogota.gov.co/sisjur/normasINorma1.jsp $? \mathrm{i}=5264$

Real Academia de la Lengua Española (2013). Diccionario de la Lengua Española Plus. Bogotá: Editorial Norma.

Recursostic. La Ciba de la Paz. Recuperado de: Antecedentes históricos del pensamiento de la cultura de la paz. http://recursostic.educacion.es/secundaria/edad/4esoetica/quincenalO/ impresos/quincena10.pdf

Sánchez, (2011). Hacia la interculturalidad de la cultura de paz. tia perspectiva educativa. Dedica. Revista de Educacao e humanidades, 1, 117-136.

Tuvilla, [(2010). Cultura de Paz. Fundamentos y claves educativas. Madrid: CCP.

Wikipedia, la enciclopedia libre. Recuperado de: La cultura de la paz: https://es.wiipedia.org/wiizCultura_de_la_paz 